\title{
Confiabilidad y validez de la Escala de Psicopatía Revisada de Hare (PCL-R) en la población carcelaria cubana
}

\author{
Piedad Medina, ${ }^{1}$ Mitchell Valdés-Sosa, ${ }^{1}$ Lídica Galán García, ${ }^{1}$ Susan Vergara Almeyda, ${ }^{2}$ Orlando Couso ${ }^{3}$
}

Artículo original

\section{SUMMARY}

The psychometric properties of the PCL-R (Psychopathy Checklist-Revised) have been standardized in numerous countries. This article evaluates if the PCL-R can be also validated in the Cuban prison population. The sample was compound by 124 males inmates, imprisoned for committing violent crimes. The PCL-R Spanish version of Moltó et al. was applied. The reliability and the internal consistency of the scale were consistent with similar investigations. A two factor structure was obtained. Factor 1 describes the Antisocial behavior and Factor 2 describes the Interpersonal-Affective traits of the scale. The two factors showed different patterns of correlation with validated instruments to measure personality traits and confirm the reliability of the construct.

Key words: Standardization, Psychopathy Checklist-Revised, validity, psychopathy, prison sample.

\section{INTRODUCCIÓN}

La psicopatía es una entidad psicopatológica compuesta por una constelación de rasgos de personalidad. Se plantea que "quizá es el más reconocido de los trastorno de personalidad" ${ }^{1-3}$ Lo cierto es que se ha descrito como el más importante constructo en el sistema criminal de justicia y a su vez como "que puede ser el concepto forense más importante de los comienzos del siglo XXI" ${ }^{4 \cdot 6}$ Estudios epidemiológicos que examinan la prevalencia de psicopatía en las muestras forenses revelan que mientras que $80 \%$ de los presos estadounidenses alcanzan el diagnóstico para el trastorno de personalidad antisocial (TPA), sólo de 15 a 25\% de ellos obtienen el criterio para psicopatía según la Psychopathy Checklist-Revised (PCL-R). ${ }^{7}$

Desde las últimas décadas, la PCL- $\mathrm{R}^{8,9}$ ha sido el instrumento de referencia para el diagnóstico de psicopatía, tanto en las poblaciones carcelarias como en las psiquiátricas fo-

\section{RESUMEN}

Las propiedades psicométricas de la Escala de Psicopatía de Hare (PCLR) han sido estandarizadas en diferentes países. Este artículo evalúa si la PCL-R puede ser validada en la población carcelaria cubana. La muestra estuvo compuesta por 124 sujetos, presos por cometer crímenes violentos, a quienes se les aplicó la adaptación española de Moltó et al. (2000). La confiabilidad y la consistencia interna de la escala fueron similares a otros estudios realizados. El análisis factorial mostró una estructura de dos factores, donde el Factor 1 representa los rasgos que describen la conducta antisocial y el Factor 2, los rasgos que describen las relaciones interpersonales y la afectividad. Los dos factores exhiben una alta correlación con distintos instrumentos validados que miden rasgos de personalidad, lo que confirma la validez del constructo.

Palabras clave: Estandarización, Psychopathy Checklist-Revised, psicópatas, validación, población carcelaria.

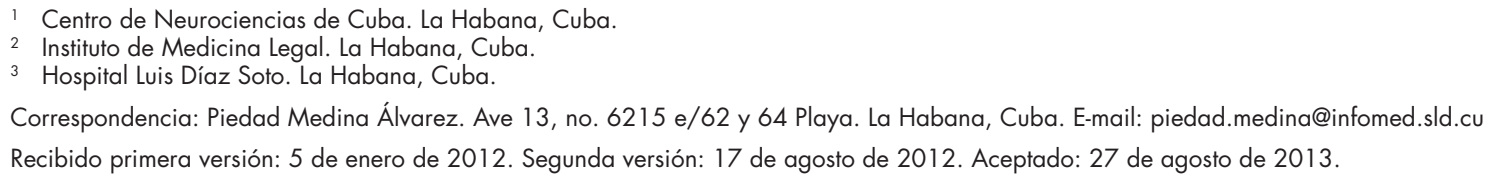




\section{MATERIAL Y MÉTODOS}

\section{Participantes}

La muestra estuvo compuesta por un total de 124 sujetos, presos por cometer crímenes violentos (asesinatos y homicidios), cuyas edades estaban comprendidas entre 18 y 62 años (media 31.14, DS=9.09). El tiempo en prisión debía ser menos de dos años y no podían tener antecedentes psiquiátricos del Eje I (basados en los criterios del SCAN versión 2.1) ni ser portadores de retraso mental en alguna de sus clasificaciones. La escolaridad se distribuyó de la siguiente forma: nivel primario 18 (14.6\%) sujetos; secundario: 70 (56.5\%); preuniversitario: 29 (23.3\%) y técnico medio: siete (5.6\%) sujetos. En cuanto al estado civil de los probandos, $74(59.7 \%)$ eran solteros, $34(27.4 \%)$ casados y $16(12.9 \%)$ se encontraban viviendo en concubinato con sus parejas.

La población estudiada en cuanto a su composición étnica estuvo constituida por $48(38.7 \%)$ sujetos afroamericanos, $42(33.9 \%)$ mestizos y $34(27.4 \%)$ caucásicos. Con base en los expedientes de los reos, se pudo clasificar a los sujetos acorde a sus antecedentes delictivos en reincidentes y sin antecedentes. De los 124 sujetos estudiados, 38 (30.6\%) ya habían estado en prisión y 86 (69.4\%) no tenían antecedentes penales.

Para iniciar la investigación se les explicó a los sujetos a evaluar los objetivos de la misma, enfatizando que tenían la opción de abandonar el estudio en cuanto lo deseasen y que los resultados de la misma no influirían en sus condenas ni apelaciones. Todos firmaron un Consentimiento informado. Se comenzó la investigación con 131 sujetos previa revisión de los expedientes penales. Durante la misma, siete de ellos la abandonaron, quedando compuesta la muestra final por 124 sujetos. Esta investigación fue dirigida y aprobada por el Comité de Ética del Centro de Neurociencias de Cuba y del Ministerio de Salud Pública.

\section{Metodología utilizada}

La evaluación neuropsiquiátrica consistió en una entrevista estructurada con datos generales, la PCL-R y la aplicación de un grupo de instrumentos que evalúan diferentes rasgos de personalidad relacionados con la psicopatía con el objetivo de estudiar la validez concurrente de la PCL-R y sus factores Interpersonal-Afectivo y la Conducta Antisocial. Éstos fueron los siguientes:

a) Cuestionario para la Evaluación Clínica en Neuropsiquiatría (SCAN, versión 2.1), ${ }^{19}$ cuestionario de psicopatología elaborado por la Organización Mundial de la Salud. Se utiliza como herramienta para el diagnóstico de enfermedades mentales en adultos.

b) Escala de Psicopatía (R) de Hare (PCL-R) ${ }^{8,9}$ Es una entrevista semiestructurada que evalúa tanto la conducta como los rasgos de personalidad afines a la psicopatía. La escala está compuesta por 20 reactivos y cada uno tie- ne una puntuación de 0 a 2 puntos $(0=$ no aplica; $1=$ aplica en ciertas circunstancias y $2=$ aplica completamente al sujeto evaluado). El valor total de la escala es de 40 puntos y el punto de corte para la psicopatía es de 30. Existen dos modelos factoriales de la escala realizada por Hare: la versión de 1991 con dos factores y la realizada en el 2003 con cuatro factores. En esta investigación se utilizará el modelo de dos factores: Factor 1 (F1) InterpersonalAfectivo; Factor 2 (F2) Conducta antisocial del sujeto.

Fueron seleccionados y entrenados para evaluar la escala seis especialistas en Psiquiatría. Se utilizó la versión de la PCL-R adaptada y validada en la población carcelaria española. ${ }^{16} \mathrm{~A}$ cada uno de los psiquiatras le correspondió evaluar un grupo determinado de sujetos. La metodología para la evaluación del instrumento indica que debe revisarse el expediente del reo que se encuentran en la prisión. A este expediente se añadieron las evaluaciones realizadas por los especialistas del grupo multidisciplinario que también participó en la investigación compuesto por psicólogos, neurofisiólogos, trabajadores sociales y reeducadores de la prisión que aportaron los resultados de sus estudios, previo a la evaluación final de la PCL-R. Esto permitió que cada psiquiatra tuviese una información objetiva e integral del individuo a evaluar.

c) Escala de Agresividad de Buss y Perry (The Aggression Questionnaire). ${ }^{20}$ (Validación española de Graña, Andreu y Peña, 2002). ${ }^{21}$ Este instrumento evalúa cuatro formas de la conducta agresiva: agresividad física, agresividad verbal, ira y hostilidad, y brinda cinco posibles respuestas que se califican de uno a cinco puntos y que oscilan desde "no me caracteriza" hasta "me caracteriza totalmente". La escala se califica de forma inversa, es decir, mientras más alta sea la puntuación del individuo, menos agresivo será. Punto de corte 77 puntos.

d) Escala del Control de los Impulsos Ramón y Cajal (ECIR y C). ${ }^{22}$ Es un instrumento que califica la conducta impulsiva en un rango de uno a cuatro puntos. La puntuación se estructura de la siguiente forma: "siempre" tres puntos, "a veces" dos puntos; "casi nunca" un punto y "nunca" cero puntos. La escala no tiene punto de corte, mientras mayor puntuación se obtenga, más impulsivo será el sujeto.

e) Escala de Autoestima de Rosenberg (The Self-Esteem Scale). ${ }^{23}$ (Se aplicó la validación española de Vázquez, Jiménez y Vázquez Morejón). ${ }^{24}$ Este instrumento consta de 10 reactivos que evalúan la autoaceptación que tiene el sujeto estudiado. La calificación va a estar estructurada en un rango de uno a cuatro puntos, donde uno punto se refiere a "muy de acuerdo", dos puntos "parcialmente de acuerdo", tres puntos "en desacuerdo", y cuatro puntos "totalmente de acuerdo". La puntuación oscila entre 10 y 40 puntos.

f) Escala de Búsqueda de Sensaciones de Zuckerman et al. (The Sensation-Seeking scale) (SSS-Form V). ${ }^{25}$ (Se aplicó 
Cuadro 1. Datos sociodemográficos de la población de psicópatas y no psicópatas

\begin{tabular}{lc}
\hline Variable & Psicópatas \\
\hline Distribución étnica & \\
- Mestizos & $45.0 \%$ \\
- Afroamericanos & $40.0 \%$ \\
- Caucásicos & $15.0 \%$ \\
Escolaridad & \\
- Primaria & $22.5 \%$ \\
- Secundaria & $62.5 \%$ \\
- Técnico medio & $2.5 \%$ \\
- Pre universitario & $12.5 \%$ \\
Reincidencia & $70.0 \%$ \\
\hline Variable & No psicópatas \\
\hline Distribución étnica & \\
- Mestizos & $28.6 \%$ \\
- Afroamericanos & $8.1 \%$ \\
- Caucásicos & $3.3 \%$ \\
Escolaridad & \\
- Primaria & $10.8 \%$ \\
- Secundaria & $53.6 \%$ \\
- Técnico medio & $7.1 \%$ \\
Reincidencia universitario & $28.6 \%$ \\
\hline
\end{tabular}

la versión española de Pérez y Torrubia). ${ }^{26}$ Es un cuestionario autoevaluado que cuenta con 40 reactivos y dos posibles respuestas: afirmativa o negativa. Este instrumento se subdivide en cuatro subescalas que evalúan di- ferentes formas de la búsqueda de sensaciones: Búsqueda de emociones (BEM), Búsqueda de excitación (BEX), Desinhibición (DIS) y Susceptibilidad al aburrimiento (SAB). No existe punto de corte; cuanto mayor puntuación, mayor búsqueda de sensaciones tiene el sujeto.

\section{Análisis estadístico}

La metodología estadística utilizada para verificar la validez y confiabilidad del constructo se basó en el cálculo del coeficiente de correlación de Pearson. Para medir la consistencia interna, se utilizó el coeficiente alpha de Cronbach. Para la estimación de los factores intrínsecos de la PCL-R, fue utilizado un modelo de análisis factorial con dos factores y se rotaron utilizando el método varimax normalizado.

\section{RESULTADOS}

\section{Análisis descriptivo de la muestra}

En la primera parte de este estudio se procedió a la aplicación de la PCL-R con el punto de corte de 30, y se clasificó la muestra en 40 (32.2\%) psicópatas y 84 (67.8\%) no psicópatas. La media y la desviación estándar de la edad en los psicópatas fue de (media=28.37; $\mathrm{DE}=6.63$ ) y en la muestra de no psicópatas (media=32.95; $\mathrm{DE}=9.82$ ). Los datos sociodemográficos de la muestra divididos en psicópatas y no psicópatas se muestran en el cuadro 1.

Cuadro 2. Estadística descriptiva para cada reactivo del PCL-R

\begin{tabular}{lcccc}
\hline Escala de Psicopatía Revisada (PCL-R) & Media & D.E. & Mediana & Rango del \\
cuartil
\end{tabular}


Por su parte, el cuadro 2 muestra la estadística descriptiva de la escala, donde la media total de la PCL-R (reflejada la mediana y los rangos de cuartiles de los reactivos) fue de 22.92 ( $\mathrm{DE}=9.90)$, siendo similar a la muestra española, $22.42,{ }^{16}$ a la del propio Hare, ${ }^{8} 23.60$ y a la muestra mexicana, $22.81 .{ }^{18}$

\section{Consistencia interna}

Para medir la confiabilidad del constructo, se utilizó el coeficiente de correlación de Pearson entre cada reactivo y la PCL-R total. El cuadro 3 muestra las correlaciones entre cada reactivo y el total de la escala y la correlación reactivototal corregida. El valor mínimo de correlaciones sin corregir obtenido fue de 0.21 y corregidas 0.13 . El punto de corte para considerar significativa la correlación con $p<0.05$ es de 0.176 .

Además se calculó el coeficiente alpha de Cronbach, el cual arrojó un valor de 0.91 y el promedio de las correlaciones inter reactivos fue de 0.38 .

\section{Análisis factorial}

Teniendo en cuenta el número de factores considerados en los estudios anteriores llevados a cabo por Moltó et al. ${ }^{16} \mathrm{y}$ Ostrosky et al. ${ }^{18}$ y el porcentaje de la varianza explicada, se consideraron dos factores. La rotación utilizada fue la varimax normalizada. El primer factor aportó $43.42 \%$ de la va-

Cuadro 3. Correlación de cada reactivo y la PCL-R Total

\begin{tabular}{lc}
\hline Escala de Psicopatía Revisada (PCL-R) & $\begin{array}{c}\text { Correlación corregida } \\
\text { entre reactivos } \\
\text { y PCL-R total }\end{array}$ \\
\hline Facilidad de palabra/encanto superficial & 0.13 \\
Sentido desmesurado de la autovalía & 0.55 \\
Necesidad de estimulación/tendencia & 0.61 \\
al aburrimiento & 0.63 \\
Mentiroso patológico & 0.72 \\
Estafador y manipulador & 0.73 \\
Ausencia de remordimiento o sentimientos & \\
de culpa & 0.79 \\
Afecto superficial y poco profundo & 0.76 \\
Insensibilidad afectiva y ausencia de empatía & 0.63 \\
Estilo de vida parasitario & 0.55 \\
Pobre autocontrol de la conducta & 0.31 \\
Conducta sexual promiscua & 0.68 \\
Problemas de conducta en la infancia & 0.67 \\
Ausencia de metas realistas a largo plazo & 0.55 \\
Impulsividad & 0.81 \\
Irresponsabilidad & 0.73 \\
Incapacidad de aceptar la responsabilidad & \\
de las propias acciones & 0.26 \\
Frecuentes relaciones maritales & \\
de corta duración & 0.56 \\
Delincuencia juvenil & 0.26 \\
Revocación de la libertad condicional & 0.65 \\
Versatilidad criminal & \\
\hline
\end{tabular}

rianza total, en tanto el segundo factor, $8.68 \%$, con lo que ambos explican un total de $52.03 \%$ de la varianza. El cuadro 4 muestra los pesos relativos de cada reactivo en ambos factores. En el Factor 1 se concentraron las características relacionadas con la conducta antisocial de los sujetos estudiados, mientras que en el Factor 2 se agrupan las alteraciones en el Factor 1 Interpersonal-Afectivo. Sólo dos reactivos, el 11 (conducta sexual promiscua) y el 17 (frecuentes relaciones maritales de corta duración), no cargaron en ninguno de los dos factores.

\section{Validez de los factores}

Con el objetivo de determinar la validez de los factores obtenidos, se correlacionó el total de la PCL-R aplicada en el estudio y los dos factores planteados con diferentes escalas, ampliamente validadas, donde cada una de ellas define rasgos afines a la psicopatía: agresividad, impulsividad, búsqueda de sensaciones y autoestima. Esto permitirá conocer la fortaleza de la PCL-R al ser validada con medidas externas para identificar de forma clínica si cada uno de los factores de la PCL-R tiene rasgos de personalidad que lo distinguen. En el cuadro 5 se presentan las correlaciones entre los factores extraídos y todas las subescalas aplicadas. La correlación con las subescalas Agresividad física, Agresividad verbal, Ira y Hostilidad fue altamente significativa e

Cuadro 4. Análisis factorial del PCL-R. Contribución de cada reactivo en los Factores 1 y 2

\begin{tabular}{|c|c|c|}
\hline \multirow[b]{2}{*}{ Reactivos del PCL-R } & \multicolumn{2}{|c|}{$\begin{array}{l}\text { Contribución } \\
\text { por reactivo }\end{array}$} \\
\hline & Factor 1 & Factor 2 \\
\hline Facilidad de palabra / encanto superficial & -0.37 & 0.63 \\
\hline Sentido desmesurado de la autovalía & 0.14 & 0.72 \\
\hline $\begin{array}{l}\text { Necesidad de estimulación/tendencia } \\
\text { al aburrimiento }\end{array}$ & 0.47 & 0.45 \\
\hline Mentiroso patológico & 0.42 & 0.58 \\
\hline Estafador y manipulador & 0.42 & 0.68 \\
\hline $\begin{array}{l}\text { Ausencia de remordimiento } \\
\text { o sentimiento de culpa }\end{array}$ & 0.54 & 0.56 \\
\hline Afecto superficial y poco profundo & 0.65 & 0.54 \\
\hline $\begin{array}{l}\text { Insensibilidad afectiva } \\
\text { y ausencia de empatía }\end{array}$ & 0.64 & 0.50 \\
\hline Estilo de vida parasitario & 0.65 & 0.27 \\
\hline Pobre autocontrol de la conducta & 0.27 & 0.58 \\
\hline Conducta sexual promiscua & 0.16 & 0.29 \\
\hline Problemas de conducta en la infancia & 0.76 & 0.24 \\
\hline Ausencia de metas realistas a largo plazo & 0.67 & 0.36 \\
\hline Impulsividad & 0.26 & 0.61 \\
\hline Irresponsabilidad & 0.70 & 0.51 \\
\hline $\begin{array}{l}\text { Incapacidad de aceptar la responsabilidad } \\
\text { de sus propias acciones }\end{array}$ & 0.43 & 0.70 \\
\hline $\begin{array}{l}\text { Frecuentes relaciones maritales } \\
\text { de corta duración }\end{array}$ & 0.01 & 0.38 \\
\hline Delincuencia juvenil & 0.80 & 0.03 \\
\hline Revocación de la libertad condicional & 0.46 & -0.06 \\
\hline Versatilidad criminal & 0.67 & 0.30 \\
\hline
\end{tabular}


Cuadro 5. Correlación entre la PCL-R total, sus factores y escalas autoaplicadas que miden personalidad

\begin{tabular}{lccc}
\hline Variables & Factor 1 & Factor 2 & $\begin{array}{c}\text { PCL-R } \\
\text { total }\end{array}$ \\
\hline Agresividad física & -.327 & -.523 & -.445 \\
& $\mathrm{p}=0.000$ & $\mathrm{p}=0.0005$ & $\mathrm{p}=0.000$ \\
Agresividad verbal & -.295 & -.469 & -.424 \\
& $\mathrm{p}=0.010$ & $\mathrm{p}=0.000$ & $\mathrm{p}=0.000$ \\
Ira & -.198 & -.411 & -.330 \\
& $\mathrm{p}=0.027$ & $\mathrm{p}=0.000$ & $\mathrm{p}=0.000$ \\
Hostilidad & -.273 & -.445 & -.408 \\
& $\mathrm{p}=0.002$ & $\mathrm{p}=0.000$ & $\mathrm{p}=0.000$ \\
Búsqueda emociones & .005 & .121 & .097 \\
& $\mathrm{n} . \mathrm{s}$. & $\mathrm{n} . \mathrm{s}$. & $\mathrm{n} . \mathrm{s}$. \\
Búsqueda excitación & .106 & .240 & .235 \\
& $\mathrm{n} . \mathrm{s}$. & $\mathrm{p}=0.007$ & $\mathrm{p}=0.009$ \\
Desinhibición & .191 & .339 & .332 \\
Susceptibilidad al aburrimiento & .272 & .390 & .349 \\
& $\mathrm{p}=0.034$ & $\mathrm{p}=0.000$ & $\mathrm{p}=0.000$ \\
Escala control impulsos & .251 & .485 & .406 \\
& $\mathrm{p}=0.005$ & $\mathrm{p}=0.000$ & $\mathrm{p}=0.000$ \\
Autoestima & .235 & .453 & .383 \\
& $\mathrm{p}=0.009$ & $\mathrm{p}=0.000$ & $\mathrm{p}=0.000$ \\
\hline & & &
\end{tabular}

inversamente proporcional con los factores Antisocial e Interpersonal-Afectivo, y con la PCL-R. La subescala de Búsqueda de emociones no obtuvo correlación significativa con ningún factor ni con la PCL-R. La Búsqueda de excitación tampoco se relacionó con el Factor 1 (Interpersonal-Afectivo), pero sí obtuvo una alta correlación tanto con el Factor 2 (Conducta antisocial), así como con el valor total de la escala mientras que la Desinhibición, Susceptibilidad al aburrimiento, el Control de impulsos y la Autoestima mostraron una alta correlación tanto con la PCL-R total como con el Factor 1 y el Factor 2.

\section{DISCUSIÓN}

La versión en español de la PCL-R aplicada en nuestra muestra de estudio fue la reportada por Moltó et al. ${ }^{16}$ en la población española. Los resultados obtenidos en este estudio son consistentes con investigaciones similares realizadas con anterioridad en diferentes poblaciones carcelarias ${ }^{8,16,18}$ en referencia a diferentes aspectos que a continuación discutiremos. En primer lugar, el porcentaje de reincidencia como variable criminológica en la muestra de psicópatas fue mayor que en la muestra de no psicópatas, lo que coincide con el estudio chileno. ${ }^{27}$ No obstante, el estudio chileno obtuvo un porcentaje superior con los psicópatas (95.4\%) que el estudio realizado por nosotros (70\%). En segundo lugar, las características psicométricas evaluadas demuestran que la versión en español de la PCL- $\mathrm{R}^{16}$ tiene una alta fiabilidad para la población carcelaria cubana de violentos, así como también replicabilidad con resultados anteriores. El tamaño de muestra utilizada es aproximadamente igual a estudios realizados con anterioridad en España, ${ }^{16}$ Argentina $^{17}$ y México. ${ }^{18}$ Los valores obtenidos de 0.91 en nuestro estudio no difieren de los obtenidos en los estudios de Moltó et al. ${ }^{16}$ (.85 para el alfa de Cronbach y .22 para la correlación interreactivos). Por otra parte, el estudio de Ostrosky y et al. ${ }^{18}$ arroja valores de .87 y .30 , y Hare $^{8}$ obtuvo .87 y .26, respectivamente. Otro hallazgo que coincide con resultados previos es que la evaluación de la escala de Hare con el punto de corte de 30 definido desde los inicios de la PCL-R para discriminar psicópatas, y no psicópatas, así como también los valores medios de la PCL-R, tuvieron una alta coincidencia con las estandarizaciones realizadas hasta el momento. ${ }^{16-18,27}$

En cuanto a la consistencia interna de la escala, coincidimos con los trabajos anteriores ${ }^{16-18,27}$ por ser similares los reactivos que se correlacionan con la PCL-R total. Sin embargo, cada estudio reporta diferentes reactivos que no tienen correlación significativa. En el caso de nuestro estudio, el reactivo 1 (Facilidad de palabra/encanto superficial) no obtuvo correlación alguna. En cambio, Moltó et al. ${ }^{16}$ informaron los reactivos cuatro y 16 , mientras que en el estudio mexicano ${ }^{18}$ todos los reactivos mostraron una alta correlación.

En el análisis factorial realizado, no todos los estudios retienen la misma cantidad de factores: tres de los estudios reportan dos factores ${ }^{8,16,18}$ y sólo dos extraen cuatro factores. ${ }^{17,27}$ El criterio que más pesó para extraer el número de factores fue la interpretación de éstos y la replicabilidad con otros estudios, así como también el porcentaje de varianza explicada. En nuestra investigación, los factores tres y cuatro sólo explicaban la influencia de los reactivos [10, 14] y [11, 17] respectivamente, con muy bajo porcentaje de variabilidad explicada, sólo se obtuvo un 10\% más al agregar ambos factores, que además no fueron reportados en los factores ya determinados en estudios anteriores. ${ }^{8,16,18}$ Los factores retenidos 1 y 2 muestran que el primero básicamente puede ser explicado por la conducta antisocial; sin embargo, se mezclan los reactivos: Afecto superficial y poco profundo e Insensibilidad afectiva y ausencia de empatía, lo que a su vez pudiera explicar que, en esta muestra, la conducta antisocial elevada es también capaz de elevar la afectividad. Una diferencia con la muestra española y el estudio de $\mathrm{Hare}^{8}$ está en el reactivo 14 (impulsividad), que obtuvo mayor peso en el factor que describe las relaciones interpersonales y la afectividad.

Las correlaciones realizadas entre la PCL-R total y sus Factores 1 y 2 con diferentes instrumentos que miden rasgos de personalidad demostraron la coincidencia del instrumento con las escalas aplicadas. Dada la alta correlación obtenida de los Factores 1 y 2 y de la PCL-R total con las escalas de Agresividad, Control de impulsos y Autoestima, se puede plantear que estos instrumentos pueden describir a la muestra en su relación con la PCL-R. Los reactivos problemas de conducta en la infancia, delincuencia juvenil y versatilidad 
criminal tienen un mayor peso en sus correlaciones con la agresividad, mientras que facilidad de palabra y encanto superficial, estafador y manipulador y necesidad de estimulación/tendencia al aburrimiento fueron los reactivos con mayor peso en la correlación con la escala de Búsqueda de sensaciones. La escala del Control de los impulsos pudiera verse presente en los reactivos impulsividad, pobre control de la conducta, incapacidad de aceptar sus propias acciones e irresponsabilidad, mientras que la escala de Autoestima se refleja en el reactivo sentido desmesurado de autovalía. En estudios realizados por diferentes investigadores se observa que Moltó et al. ${ }^{16}$ encontraron que las escalas autoaplicadas se correlacionaron de forma moderada con el Factor 2 mientras que el Factor 1 sólo tuvo correlación con un instrumento. Por su parte, Ostrosky et al. ${ }^{18}$ demostraron que el Factor 1 fue el más correlacionado.

Para concluir, se pudo demostrar que la PCL-R es un instrumento que tiene validez en la subpoblación carcelaria cubana compuesta por presos por delitos violentos y que cada factor de la escala tiene características propias que caracterizan el comportamiento de psicópatas y no psicópatas.

\section{REFERENCIAS}

1. Trull TJ, Durrett CA. Categorical and dimensional models of personality disorder. Ann Rev Clin Psychology 2005;1:355-380.

2. Neumann CS, Hare RD, Newman JP. The super-ordinate nature of psychopathy. J Pers Disord 2007;21:102-117.

3. Lykken DT. Psychopathy personality: The scope of the problem. En: Patrick CJ (ed.). Handbook of psychopathy New York: Guilford Press; 2006; pp. 3-13.

4. Hare RD. Psychopathy: A clinical construct whose time has come. Crim Just Behav 1996;23:25-54.

5. Monahan J, Steadman HJ, Appelbaum PS et al. The classification of violence risk. Behav Sci Law 2006;24:721-730.

6. Torrubia R, Cuquerella A. Psicopatía: una entidad clínica controvertida pero necesaria en psiquiatría forense. Rev Esp Med Legal 2008;34:25-35.

7. Hare RD: Psychopathy and risk for recidivism and violence. Criminal justice, mental health, and the politics of risk. London: Cavendish; 2004; pp. 27-47.

8. Hare RD. The Hare Psychopathy Checklist-Revised. Toronto, ON: Multi-Health Systems; 1991.
9. Hare RD. Manual for the Hare Psychopathy Checklist-Revised. Segunda edición. Toronto: Multi-Health Systems; 2003.

10. Cooke DJ, Michie C, Hart SD, Clark D. Assessing psychopathy in the United Kingdom. Concern about cross-cultural generalisability. Br J Psychiatry 2005a;186:339-345.

11. Hicks BM, Patrick CJ. Psychopathy and negative emotionality: Analyses of suppressor effects reveal distinct relations with emotional distress, fearfulness, and anger-hostility. J Abnorm Psychol 2006;115:276-287.

12. Lynam DR, Widiger TA. Using a general model of personality to identify the basic elements of psychopathy. J Person Disord 2007;21:160-178.

13. Bishop D, Hare RD. A multidimensional scaling analysis of the hare PCL-R: Unfolding the structure of psychopathy. Psicol Crime Law 2008;14:117-132.

14. Hare RD. Psychopathy: A clinical and forensic overview. Psychiatr Clin N Am 2006;29:709-724.

15. Cooke DJ, Michie C. An item response theory evaluation of Hare's Psychopathy Checklist. Psych Assess 1997;9:2-13.

16. Moltó J, Poy R, Torrubia R. Standardization of the Hare Psychopathy Checklist-Revised in Spanish prison sample. J Pers Disord 2000;14: 84-96.

17. Folino JO, Hare RD. Listado revisado para la verificación de la psicopatía: su estandarización y validación en Argentina. Acta Psiquiat Psicol Am L 2005;51:90-104.

18. Ostroski-Solís F, Ruiz Ortega A, Arias García, N, Vázquez Vera V. Estandarización de la PCL-R en población penitenciaria mexicana. Revista Neuropsicología, Neuropsiquiatría Neurociencias 2008;8:49-58.

19. SCAN: Cuestionario para la Evaluación Clínica en Neuropsiquiatría. Madrid: OMS; 1992.

20. Buss A, Perry M. The Agression Questionnaire. J Pers Soc Psychol 1992;63:3.

21. Graña JL, Andreu JM, Peña ME. Adaptación psicométrica de la versión española del cuestionario de agresión. Psicothema 2002;14:476-482.

22. Ramos Brieva JA, Gutiérrez Zotes A, Saíz Ruíz J. Escala de Control de los Impulsos "Ramón y Cajal" (ECIR y C). Act Esp Psiquiatr 2002;30:160-174.

23. Rosenberg M. Self-esteem scale society and adolescent self image. Princeton, NJ: Princeton University Press; 1965.

24. Vázquez J, Jiménez R, Vázquez-Morejón R. Escala de autoestima de Rosenberg: fiabilidad y validez en población clínica española. Apuntes Psicología 2004;22:247-255.

25. Zuckerman M, Eysenck SBG, Eysenck HJ. Sensation-seeking in England and America: Cross-cultural, age, and sex comparisons. J Consult Clin Psychol 1978;46:139-149.

26. Pérez J, Torrubia R. Fiabilidad y validez de la versión española de la escala de búsqueda de sensaciones (forma V). Rev Latinoam Psicol 1986;18:7-22.

27. León-Mayer E, Asún-Salazar D, Folino JO. Confiabilidad y validez de la versión chilena de la Hare PCL-R. Rev Fac Med 2010;58:103-114.

Artículo sin conflicto de intereses 